\title{
Comprehensive Appraisal of Human Capital in Northern Anhui Province Based on Improved Entropy Method
}

\author{
G. HE \& L. YANG \\ School of economics \& management, Anhui University of Science \& Technology, Huainan, Anhui, China
}

\begin{abstract}
For a region with a population of over 30 million, quality of human capital in northern Anhui province plays a pivotal role in the development of the region and even the whole province. Based on the connotation of human capital, we found it is evaluated by improved entropy method from three aspects, which are cultural \& sports, research \& development and physical function \& quality of life. The result demonstrates that knowledge, potential and physical qualities have the same effect on human capital. Also, compared with the size of population, the effect of quality of human capital on economic development is more crucial. Comparing with the size of population, additionally, quality of human capital is a key decision for advance. KEYWORD: Human capital; Evaluation index; Improved entropy method
\end{abstract}

Human resource includes the quantity and quality of labor force, while human capital, emphasizing on the quality, reflects knowledge, skills and health status of workers [1]. A large number of data shows that human capital plays a decisive role in the sustainable development of economy, culture and society. For over 30 billion people in the six cities of the northern Anhui province, there is a definite link between the development of the whole Anhui province and the situation of human capital quality of them.

\section{LITERATURE REVIEW}

At present, domestic experts and scholars have made certain achievements on the comprehensive evaluation of the regional human capital. In 2006, Sun Licheng and Li Qun analyzed the mufti-index comprehensive appraisement of the capital of talents quality of the 31 zones of China in 2003 by attribute theory model. These zones were divided into three levels, i.e. superior, medium, and inferior, and zones in the same level were sorted by confidence [2]. Yue Shujin in 2008 considered that the comprehensive index of human capital in China appeared unbalanced and regional, and index of individual human capital investment would widen the gap between comprehensive human capital indexes [3]. In 2011, Lu Yuanquan and Ma Leixin calculated the overall score of 31 provinces of China human resource situation by factor analysis and divided these provinces into four categories based on their characteristics [4]. Li Yumeng (2012) believed that strengthening education, increasing employment channels, optimizing social environment, raising health care's quality and changing the traditional ideas were required to promote Qinghai rural female human capital quality [5].

Taken together, methods of existing studies include attribute theory model, factor analysis, etc. These index weights are far from complete and subjectivity relatively strong. However, improved entropy method reflects how the index affects the study results and maintains the objectivity of the weights at utmost, which is a more suitable method to evaluate the situation of human capital. The population of Northern Anhui is accounted for more than $50 \%$ of the whole province. So the effect of its quality of the human capital on the development of the whole Anhui province can not be ignored. It is necessary to research on quality of the human capital of Northern Anhui.

\section{CONSTRUCTION OF INDEX SYSTEM}

Evaluation indicators of human capital are selected on the basis insisting on principles such as scientificity, operability, integrity, generality, etc. The evaluation index system including 3 first grade indices (Cultural \& Sports, Research \& Development, Physical Function \& Quality of Life) and 15 second grade indices is founded after been 
researched on diversity and complexity of the impact factor of human capital and combined with its connotation and the existing research results (see table 1).

Table 1. Evaluation index of human capital of six cities in Northern Anhui

\begin{tabular}{|c|c|}
\hline \multicolumn{2}{|r|}{ Index } \\
\hline I & II \\
\hline Cultural \& Sports & $\begin{array}{l}\text { Number of sports and cultural venues }{ }^{*} \text { ( / million people) } \\
\text { Volume of books per capita } \\
\text { Illiteracy rate }(\%) \\
\text { Average education attainment(years) } \\
\text { Investment in education(million) }\end{array}$ \\
\hline Research \& Development & $\begin{array}{l}\text { R\&D ful-time equivalent (manyr/million people) } \\
\text { Number of professionals ( /million people) } \\
\text { Number of effective invention patents ( / million people) } \\
\text { Number of scientific papers ( / million people) } \\
\text { Number of scientific books ( / million people) }\end{array}$ \\
\hline Physical Function \& Quality of Life & $\begin{array}{l}\text { GDP per capita } \\
\text { Proportion of labor force }{ }^{* *}(\%) \\
\text { Level of gender balance }^{* * *} \\
\text { Nutritional status } \\
\text { Number of doctors (/ million people) }\end{array}$ \\
\hline
\end{tabular}

Notes:

*Number of sports and cultural venues: the sum of the number of the stadiums and the theaters;

** Proportion of labor force: the population aged 15-59 per the city's total population;

*** Level of gender balance: number of male set the female number is 100 (the closer to 100 the better);

****Nutritional status: proportion of consumer expenditure of grain and edible oil per consumer expenditure of food. In general, the lower proportion of consumer expenditure of grain and edible oil per consumer expenditure of food is, the higher nutritional value food is so that health will be the better, and vice versa.

\section{IMPROVED ENTROPY METHOD}

The collected data is handled by improved entropy method [6], which is from Anhui Statistical Yearbook (2014) [7]. All evaluation index with objective weight can be entrusted by the entropy method, that is to say, individual subjective factors are avoided in the decision process of weights of evaluation factors. Due to the log and entropy are applied in the process of calculating and extreme and negative are not directly involved in the calculation, the entropy method has to be improved. Presently, there are two kinds of improved method: effect function method and standard transform method. And the latter without relying on subjective information, minimizes the effect of extreme value on the result, which is more in line with the research requirements. Therefore, standard transform method will be adopted to handle data in this paper.

\subsection{Data standardization}

Before being handled, the data need to be purified dimensionless. The Z-score standardization method makes selected data fit the standardized normal distribution by SPSS 20.0. where $x_{i j}^{\prime}=\operatorname{standardized} x_{i j} ; x_{i j}=$ index $\mathrm{j}$ of city $i$; $\bar{x}_{j}=$ the average of index $j ; \sigma_{j}=$ the standard deviation of index $j$.

$$
x_{i j}^{\prime}=\left(x_{i j}-\bar{x}_{j}\right) / \sigma_{j}
$$

\subsection{Improvement of index}

For ensuring the negative data is eliminated after standardization, the data needs to be improved. That means $x_{i j}^{\prime}$ should be translated $k$ value to generate a new coordinate $x_{i j}^{\prime \prime}$

$$
x_{i j}^{\prime \prime}=x_{i j}^{\prime}+k
$$

\subsection{Calculation of proportion $R_{i j}$}

$$
R_{i j}=x_{i j}^{\prime \prime} / \sum_{i=1}^{m} x_{i j}^{\prime \prime}
$$

3.4 Calculation of entropy $e_{j}\left(e_{j} \in[0,1]\right)$

$$
e_{j}=-\left(\frac{1}{\ln m}\right) \sum_{i=1}^{m} R_{i j} \ln R_{i j}
$$


3.5 Calculation of difference coefficient $y_{j}$

$y_{j}=1-e_{j}$

3.6 Calculation of weight $w_{j}$

$$
w_{j}=\frac{y_{j}}{\sum_{j=1}^{n} y_{j}}(j=1,2, \cdots, n)
$$

3.7 Calculation of scores $w_{j}$ of human capital by weighted averages method

$P_{i}=\sum_{j=1}^{n} w_{j} R_{i j}$

\section{EVALUATION OF HUMAN CAPITAL QUALITY}

Combining with Anhui Statistical Yearbook (2014) human capital quality of six cities in Northern Anhui is analyzed.

\subsection{Weight of indexes}

Weight of indexes is calculated by improved entropy method (see table 2).
Table 2 Index weight of evaluation system on human capital of six cities in Northern Anhui

\begin{tabular}{|l|c|}
\hline Index & Weight \\
\hline Cultural \& Sports & $\mathbf{0 . 3 2 4 9}$ \\
Number of sports and cultural venues & 0.0627 \\
( / million people) & 0.0585 \\
Volume of books per capita & 0.0695 \\
Illiteracy rate (\%) & 0.0702 \\
Average education attainment (years) & 0.0640 \\
Investment in education(million) & $\mathbf{0 . 3 2 7 5}$ \\
\hline Research \& Development & 0.0698 \\
R\&D full-time equivalent & \\
(manyr/million people) & 0.0646 \\
Number of professionals & \\
( /million people) & 0.0628 \\
Number of effective invention patents & \\
( / million people) & 0.0661 \\
Number of scientific papers & \\
( / million people) & 0.0642 \\
Number of scientific books & $\mathbf{0 . 3 4 7 6}$ \\
( / million people) & 0.0704 \\
\hline Physical Function \& Quality of Life & 0.0767 \\
GDP per capita & 0.0686 \\
Proportion of labor force (\%) & 0.0656 \\
Level of gender balance & 0.0663 \\
\hline Nutritional status & \\
Number of doctors ( / million people) & \\
\hline
\end{tabular}

\subsection{Score and rank}

The score and rank of the six cities are gained by the weight in table 1 (see table 3 ).

Table3 Score and rank of human capital quality of six cities in Northern Anhui

\begin{tabular}{|c|c|c|c|c|c|c|c|c|}
\hline \multirow{2}{*}{ City } & \multicolumn{2}{|c|}{ Cultural \& Sports } & \multicolumn{2}{c|}{ Research \& Development } & \multicolumn{2}{c|}{$\begin{array}{c}\text { Physical Function \& } \\
\text { Quality of Life }\end{array}$} & \multicolumn{2}{c|}{ Overall Merit } \\
\cline { 2 - 9 } & Score & Rank & Score & Rank & Score & Rank & Score & Rank \\
\hline Huaibei & 0.0698 & 2 & 0.0596 & 3 & 0.0756 & 2 & 0.2050 & 3 \\
\hline Bozhou & 0.0381 & 6 & 0.0315 & 6 & 0.0290 & 6 & 0.0986 & 6 \\
\hline Suzhou & 0.0411 & 4 & 0.0325 & 5 & 0.0512 & 4 & 0.1248 & 4 \\
\hline Bengbu & 0.0612 & 3 & 0.0824 & 2 & 0.0675 & 3 & 0.2110 & 2 \\
\hline Fuyang & 0.0393 & 5 & 0.0344 & 4 & 0.0440 & 5 & 0.1177 & 5 \\
\hline Huainan & 0.0755 & 1 & 0.0871 & 1 & 0.0803 & 1 & 0.2429 & 1 \\
\hline
\end{tabular}

\subsection{Analysis}

Due to data has been manipulated positively, the higher the score is, the better the part is. Namely, the higher the score of Overall Merit is, the better the human capital quality is. Then the six cities could be divided into three levels by the score of Overall Merit in table 3.

Huainan is the first level city. It scores better than others in all three parts, so it scores in Overall Merit far more than the other five cities. All in all, Huainan is better at cultivating talents and fulfilling their potential comparing the others, which promotes the development of economy and society.

Bengbu and Huaibei belong to the second level. The scores in the three parts and Overall Merit of the two cities are all upper-middle in table 3. Huaibei has a little advantage of Cultural \& Sports and Physical Function \& Quality of Life, but its score of Research \& Development is much lower than that of Bengbu, which causes its score in Overall Merit lower than that of Bengbu. 
The cities in the last level are Suzhou, Fuyang and Bozhou. Their scores in all three parts and Overall Merit are all in down level. Especially, scores of Bozhou in all events rank at the bottom, and are far more below the top city Huainan. In summary, the three cities have the toughest challenge on human capital development in Northern Anhui.

\section{CONCLUSION}

There are several conclusions after analyzing human capital quality of six cities in Northern Anhui.

First of all, knowledge, potential and physical quality have the same effect on human capital quality. Because the three first class indexes have approximately the same weight: $0.3249,0.3275$ and 0.3476 respectively. So to improve the human capital quality learning and using existed knowledge and technology is not only to be considered, but fostering creative ability is also an indispensable part. Moreover, improving the level of fitness should been paid attention. Good health, after all, is the cornerstone of everything.

In the second place, quality, compared with quantity, of human capital is the key to determine the economic development. In practical terms, GDP figures of the last three cities are higher than the first three cities slightly, but their population arrives at 4 million even 7 million, which is almost twice as many as the first three cities' 2 or 3 million. Hence one can see that, the positive effect of human capital quality on economy is more than quantity does. Therefore, the government of Northern Anhui should control the size and raise the general quality of the population.

Above all, human capital qualities of six cities in Northern Anhui have clearly diverged. So cities with higher human capital quality should strengthen independent inventive capability and the absorption of imported technology under optimizing the population structure. Additionally, it is up to these cities to show leadership to help cities with low advance of human capital. And relatively backward cities ought to increase input in education and control the population size to raise basic technological literacy and skills in the population and put individuals' ability into full play. Besides, cities with lower human capital quality could try harder to import talents and establish long-term mechanism to improve human capital quality.

\section{ACKNOWLEDGEMENTS}

This paper is supported by fund of humanities and social-science project of Anhui college (SK2014ZD024) and humanities and social science fund plan of the ministry of education (14YJAZH029).

\section{REFERENCES}

[1] Sun,Z.F. 2009. Analysis of human resources in transformation of economic growth. Tongji University Journal Social Science Section 11(05): 105-111.

[2] Sun,L.C. \& Li,Q. 2006. The application of attribute theory model in the comprehensive appraisement of the capital of talents quality. Mathematics in Practice and Theory 08(9): 62-69.

[3] Yue,S.J. 2008. Comprehensive evaluation and dynamic evaluation of human capital in China's provincial areas. Modern Management Science 36(4):36-37.

[4] Lu,Y.Q. \& Ma,L.X. 2011. A comparative study of human resource situation on 31 provinces of China. Statistics and Decision 12(4): 75-78.

[5] Li,Y.M. 2012. Status and development countermeasures of Qinghai rural female human resources quality. Forward Position 06(20):100-102.

[6] Sun,L.J. \& Xin,X.J. 2010. Improvement of the entropy weighting method. Statistics and Decision 16(21):153-154.

[7] Statistics Bureau of Anhui Province. 2014. Anhui Statistical Yearbook(2014). Beijing: China Statistics Press. 